\title{
Identification of alcohol stress tolerance genes of Synechocystis sp. PCC 6803 using adaptive laboratory evolution
}

\author{
Takuya Matsusako, Yoshihiro Toya, Katsunori Yoshikawa and Hiroshi Shimizu* (0)
}

\begin{abstract}
Background: Synechocystis sp. PCC 6803 is an attractive organism for the production of alcohols, such as isobutanol and ethanol. However, because stress against the produced alcohol is a major barrier for industrial applications, it is highly desirable to engineer organisms with strong alcohol tolerance.

Results: Isobutanol-tolerant strains of Synechocystis sp. PCC 6803 were obtained by long-term passage culture experiments using medium containing $2 \mathrm{~g} / \mathrm{L}$ isobutanol. These evolved strains grew on medium containing $5 \mathrm{~g} / \mathrm{L}$ isobutanol on which the parental strain could not grow. Mutation analysis of the evolved strains revealed that they acquired resistance ability due to combinatorial malfunctions of sIr1044 (mcpA) and slr0369 (envD), or slr0322 (hik43) and envD. The tolerant strains demonstrated stress resistance against isobutanol as well as a wide variety of alcohols such as ethanol, $n$-butanol, and isopentanol. As a result of introducing an ethanol-producing pathway into the evolved strain, its productivity successfully increased to $142 \%$ of the control strain.
\end{abstract}

Conclusions: Novel mutations were identified that improved the stress tolerance ability of various alcohols in Synechocystis sp. PCC 6803.

Keywords: Synechocystis sp. PCC 6803, Alcohol stress tolerance, Adaptive laboratory evolution, Isobutanol

\section{Background}

Cyanobacteria are promising industrial microorganisms for bio-production because the cells can directly fix atmospheric carbon dioxide and convert it to a target compound using energy from photosynthesis. In recent years, the cyanobacterial production of alcohols, such as ethanol, $n$-butanol, and isobutanol, as an alternative to petroleum has attracted attention [1]. Isobutanol is an attractive fuel because of its higher energy density and lower hygroscopicity than ethanol. Overexpression of ribulose 1,5-bisphosphate carboxylase/oxygenase genes has led to the production of $450 \mathrm{mg} / \mathrm{L}$ isobutanol production in Synechococcus elongatus PCC 7942 [2]. However, its productivity is still lower than that of fermentation

\footnotetext{
*Correspondence: shimizu@ist.osaka-u.ac.jp

Department of Bioinformatic Engineering, Graduate School

of Information Science and Technology, Osaka University, 1-5 Yamadaoka,

Suita, Osaka 565-0871, Japan
}

microorganisms such as Escherichia coli and Corynebacterium glutamicum $[3,4]$. Alcohol stress is one of the causes of low productivity in cyanobacteria. In isobutanol production of Synechocystis sp. PCC 6803, production of biomass and isobutanol increased about 1.2 and 2.5 times, respectively, by in situ removal of the produced isobutanol using a solvent trap [5]. Because the growth reduction caused by alcohol stress occurs at a low concentration of $200 \mathrm{mg} / \mathrm{L}$ isobutanol, it is highly desirable to engineer cyanobacterial strains with strong alcohol tolerance for industrial production of isobutanol.

In a previous study, transcriptome responses of Synechocystis sp. PCC 6803 to 1-butanol identified candidate genes for enhancing 1-butanol tolerance, and the expression of small heat-shock protein HspA (sll1514) and hypothetical protein CccS (slr1617) successfully improved 1-butanol tolerance [6]. Furthermore, screening from the response regulator library revealed that sll0039 and slr1037 are involved in 1-butanol tolerance in 
Synechocystis sp. PCC 6803 [7, 8]. The co-overexpression of slr1037 and sll0039 improved 1-butanol tolerance by $133 \%$ [9]. It has also been reported that an overexpression of sigB, which encoding RNA polymerase sigma factor, improved 1-butanol tolerance [10].

Adaptive laboratory evolution (ALE) is a method for strain engineering to provide tolerance against a stress condition [11, 12]. During ALE, cells are repeatedly subcultured for prolonged periods under stress conditions leading to a reduced growth rate. This allows the selection of mutants with improved growth phenotypes under the stress environment [13]. Compared to random mutagenesis approaches using mutagens such as chemicals and ultraviolet light, ALE with sequential serial passages is a relatively easy method for identifying key mutations related to the improvement because of its low mutation frequency [14]. The productivity of the target compound can be enhanced by introducing the identified mutations that improve the tolerance of the strain into an engineered producer. Because a complete genome sequence of Synechocystis sp. PCC 6803 has already been determined [15], mutation positions are easily identified by genome resequencing analysis, which aligns genomic reads of the evolved strain to a reference genome [16]. The mutations for improving acid stress tolerance in Synechocystis sp. PCC 6803 were obtained through ALE experiments under low $\mathrm{pH}$ conditions and by whole genome resequencing analysis [17].

It has been reported that cells acquiring a stress tolerance exhibit a tolerance to the same type of stress $[18,19]$. In the present study, we aimed to obtain a tolerant strain of Synechocystis sp. PCC 6803 against various types of alcohols using ALE with medium containing isobutanol, which has strong toxicity. Mutations of evolved strains were identified by whole genome resequencing and were introduced into the genome of the parental strain to evaluate the contribution for alcohol tolerance by a reverse genetics approach. We demonstrated that the acquired resistance ability of the evolved tolerant strain enhances ethanol production.

\section{Results}

\section{Adaptive laboratory evolution of Synechocystis sp. PCC} 6803 under isobutanol stress conditions

Isobutanol-tolerant strains were obtained via long-term passage culture experiments. Prior to passage cultures, Synechocystis sp. PCC 6803 was cultured under different isobutanol concentrations $(0,1,2,3$, and $5 \mathrm{~g} / \mathrm{L})$ to determine the appropriate concentration for ALE. The $\mathrm{OD}_{730}$ at $72 \mathrm{~h}$ decreased by one-half in medium containing $2 \mathrm{~g} / \mathrm{L}$ isobutanol compared to samples under no isobutanol condition (Additional file 1: Fig. S1). Passage cultures were performed using medium containing $2 \mathrm{~g} / \mathrm{L}$ isobutanol. Because various evolved strains with different mutations can be obtained in ALE experiments, four independent passage cultures were performed. The relationship between the specific growth rate and accumulated culture time during ALE experiments are shown in Additional file 1: Fig. S2. The specific growth rates obviously increased until $500 \mathrm{~h}$ in the all four cultures. Although cell aggregation was observed until $300 \mathrm{~h}$, cells eventually separated. Because no increase in the specific growth rate was observed after $1000 \mathrm{~h}$, the evolved cells at $1824 \mathrm{~h}$ were stored at $-80{ }^{\circ} \mathrm{C}$. Four strains, named $\mathrm{T}(1), \mathrm{T}(2), \mathrm{T}(3)$, and $\mathrm{T}(4)$, were isolated from the stored cultures of Nos. 1, 2, 3, and 4, respectively.

To evaluate the isobutanol tolerance of the four evolved strains obtained from the ALE experiments, the parental and the evolved strains were cultured at different isobutanol concentrations $(0,1,2,3$, and $5 \mathrm{~g} / \mathrm{L})$. The specific growth rate of the evolved strains increased compared to that of the parental strain with more than $2 \mathrm{~g} / \mathrm{L}$ isobutanol (Fig. 1). Furthermore, the evolved strain grew in the presence of $5 \mathrm{~g} / \mathrm{L}$ isobutanol which completely inhibited the growth of the parental strain. Although cell aggregation was observed in the parental strain, this was not observed in the evolved strains exposed to $2 \mathrm{~g} / \mathrm{L}$ isobutanol (Fig. 2 and Additional file 1: Fig. S3).

\section{Whole genome resequencing of the evolved strains}

To investigate the mutations within the evolved strains, whole genome resequencing was performed using a MiSeq sequencer through paired-end sequencing with a read length of $250 \mathrm{bp}$. Coverage depth was approximately 100-200 reads. Identified mutations of the evolved strains are summarized as a Venn diagram in Fig. 3. The

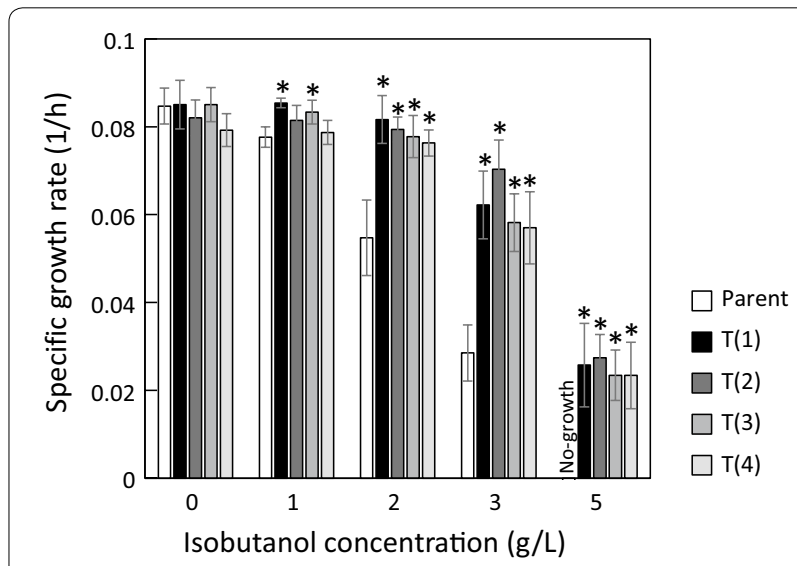

Fig. 1 Specific growth rates of the parental and the evolved strains under different concentrations of isobutanol. The asterisks indicate significant differences between the parental and the evolved strains (t test, $P<0.05)$. The specific growth rate was calculated from an

$\mathrm{OD}_{730}$ of 0 and $48 \mathrm{~h}$ of each batch culture. Error bars represent standard deviations of three replicates 


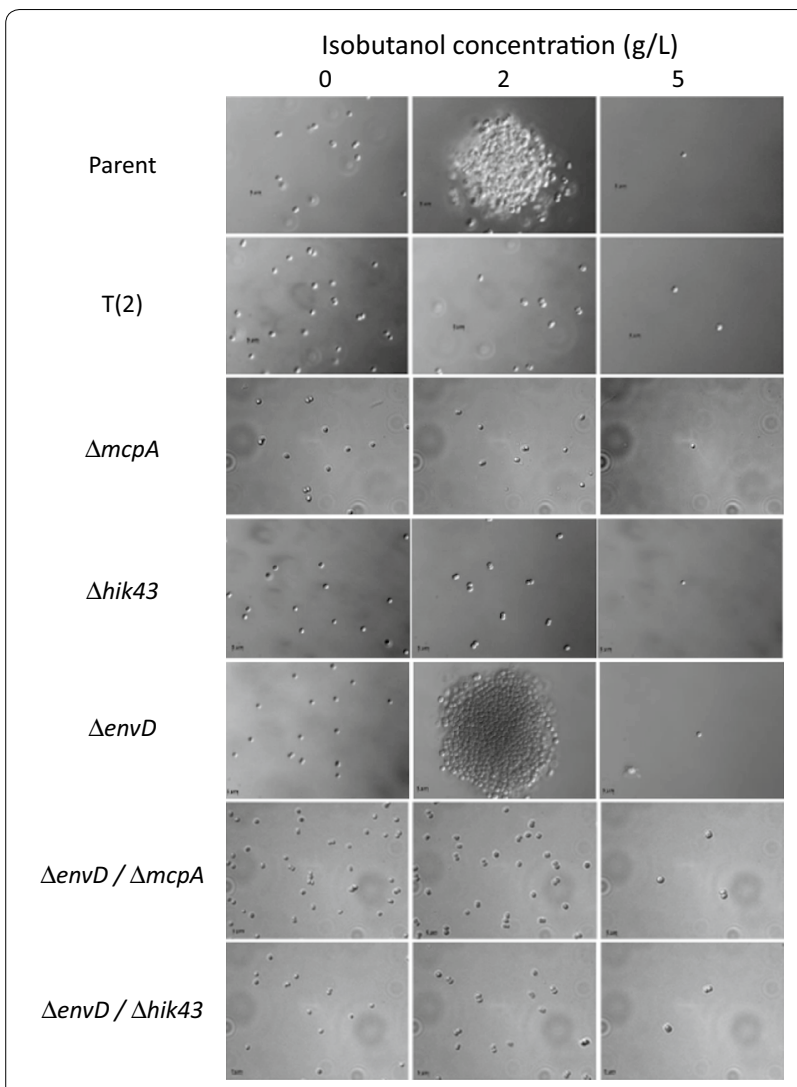

Fig. 2 Cell flocculation of parental, evolved, and knockout strains at different isobutanol concentrations $(0,2$ and $5 \mathrm{~g} / \mathrm{L})$

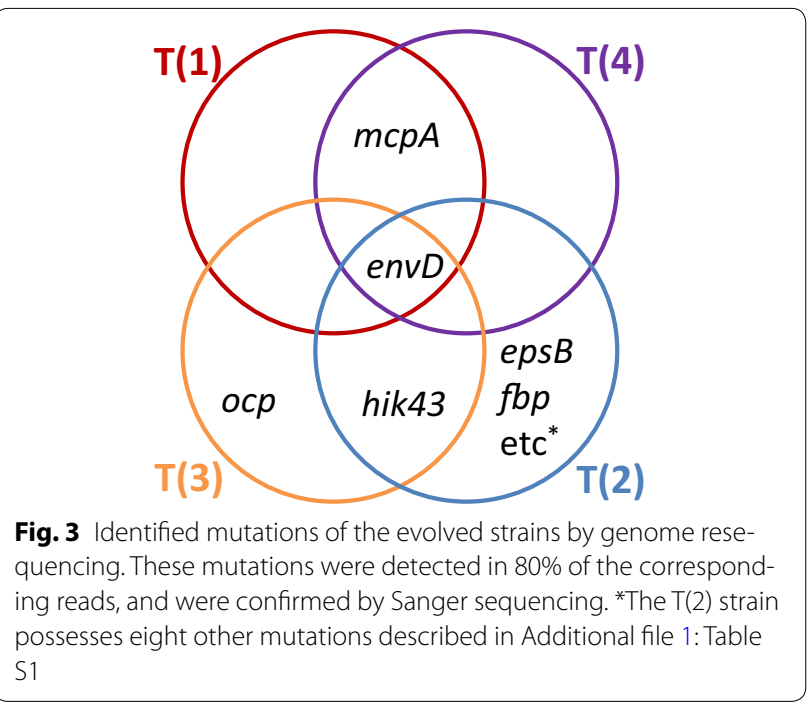

numbers of mutations in $\mathrm{T}(1), \mathrm{T}(2), \mathrm{T}(3)$, and $\mathrm{T}(4)$ strains were $2,12,2$, and 2 , respectively. $\mathrm{T}(1)$ and $\mathrm{T}(4)$ strains possessed mutations in $m c p A$, whereas $\mathrm{T}(2)$ and $\mathrm{T}(3)$ strains possessed mutations in hik43. All four evolved strains possessed mutations in $e n v D$. No mutation was identified in non-cording regions. Detailed information about the mutations is summarized in Fig. 3 and Table 1. Because the $\mathrm{T}(2)$ strain possessed a stop codon mutation in $m u t S$, which encodes DNA mismatch repair protein, a higher number of mutations were accumulated during ALE than that in other evolved strains. It has been reported that $m c p A$ and hik43 are involved in the biogenesis of pili for generating motile force [20], and envD is involved in the transport of toxin [21]. The genome resequencing results suggest that these common mutations of $m c p A$, hik43, and envD in the evolved strains could be related to the observed enhanced isobutanol tolerance.

Because the mutations of $m c p A$ in $\mathrm{T}(1)$ and $\mathrm{T}(4)$ strains lead to a frame shift and a premature stop codon, the resulting putative truncated products are 75 and 30 amino acid residues, respectively, out of 869 amino acid residues. These truncated products must have no function due to their short length. The mutation of hik43 in $\mathrm{T}(2)$ also lead to a frame shift and a premature stop codon. As the result, the putative truncated product is only 252 amino acid residues out of 1095 amino acid residues. The mutation of envD in T(1) also lead to a frame shift and a premature stop codon. As the result, the putative truncated product is only 158 amino acid residues out of 1083 amino acid residues. On the other hands, the putative products of EnvD in $\mathrm{T}(2), \mathrm{T}(3)$, and $\mathrm{T}(4)$ strains had only few amino acid substitutions.

\section{Effect of mutations on isobutanol tolerance}

To evaluate the effect of the mutations in $m c p A$, hik43, and $e n v D$ of the evolved strains on isobutanol tolerance, the mutations and corresponding gene deletions were introduced into the genome of the parental strain.

$m c p A$ encodes a methyl-accepting chemotaxis protein. No growth recovery was observed by introducing the $m p c A$ deletion in the presence of $2 \mathrm{~g} / \mathrm{L}$ isobutanol (Additional file 1: Fig. S4). Cell aggregation, which was observed in the parental strain, was dispersed in the $\triangle m c p A$ strain (Fig. 2 and Additional file 1: Fig. S5). This indicates that $m c p A$ contributes to cell aggregation under the isobutanol stress condition. The strain did not grow in the presence of $5 \mathrm{~g} / \mathrm{L}$ isobutanol, as well as its parental strain. The mcpA-R1 and mcpA-R4 strains were constructed by introducing $m c p A$ mutations derived from the $\mathrm{T}(1)$ and $\mathrm{T}(4)$ strains, respectively, into the $\triangle m c p A$ strain. Because the mcpA-R1 and mcpA-R4 strains showed a similar phenotype as the $\triangle m c p A$ strain, the $m c p A$ mutations were considered loss-of-function mutations.

hik43 encodes a histidine kinase, which is a member of the two-component hybrid sensor and regulator. Because stop codon mutations occurred in hik43 of the 
Table 1 Details about the mutations of evolved strains

\begin{tabular}{|c|c|c|c|c|c|c|c|c|}
\hline Strain & Type $^{a}$ & $\begin{array}{l}\text { Posi- } \\
\text { tion }\end{array}$ & Reference & Mutation & Gene & Type & $\begin{array}{l}\text { Mutation ratio } \\
(\%)^{\mathbf{b}}\end{array}$ & Function \\
\hline \multirow[t]{2}{*}{$\mathrm{T}(1)$} & Del & 492349 & ATCCTA & $-\mathrm{T}----$ & slr1044 (mcpA) & $\begin{array}{l}\text { Frameshift, stop } \\
\text { codon }\end{array}$ & 95 & $\begin{array}{l}\text { Methyl-accepting } \\
\text { chemotaxis protein }\end{array}$ \\
\hline & Ins & 2364114 & CCG & CCGA & slr0369 (envD) & $\begin{array}{l}\text { Frameshift, stop } \\
\text { codon }\end{array}$ & 94 & $\begin{array}{l}\text { RND multidrug efflux } \\
\text { transporter }\end{array}$ \\
\hline \multirow[t]{11}{*}{$\mathrm{T}(2)$} & Ins & 345787 & TGG & TGGG & sll0923 (epsB) & $\begin{array}{l}\text { Frameshift, stop } \\
\text { codon }\end{array}$ & 92 & Unknown protein \\
\hline & Ins & 389860 & $\mathrm{CCC}$ & CCCC & sll1025 & $\begin{array}{l}\text { Frameshift, stop } \\
\text { codon }\end{array}$ & 86 & Hypothetical protein \\
\hline & Ins & 549361 & CGG & CGGG & sll1636 (fbp) & $\begin{array}{l}\text { Frameshift, stop } \\
\text { codon }\end{array}$ & 84 & $\begin{array}{l}\text { Ferripyochelin-binding } \\
\text { protein }\end{array}$ \\
\hline & SNP & 755131 & CTG & TTG & sll1924 (sycrp2) & Leu $\rightarrow$ Leu & 99 & CAMP receptor protein \\
\hline & Ins & 1041211 & GGG & GGGG & $\operatorname{sir1233(frdA)}$ & $\begin{array}{l}\text { Frameshift, stop } \\
\text { codon }\end{array}$ & 94 & $\begin{array}{l}\text { Succinate dehydro- } \\
\text { genase flavoprotein } \\
\text { subunit }\end{array}$ \\
\hline & Del & 2270044 & GGG & $-G G$ & slr0322 (hik43) & $\begin{array}{l}\text { Frameshift, stop } \\
\text { codon }\end{array}$ & 93 & $\begin{array}{l}\text { Two-component } \\
\text { hybrid sensor and } \\
\text { regulator }\end{array}$ \\
\hline & SNP & 2279149 & CAG & GAG & slr0326 & $\mathrm{Gln} \rightarrow \mathrm{Glu}$ & 97 & Hypothetical protein \\
\hline & SNP & 2364333 & GTT & GGT & envD & $\mathrm{Val} \rightarrow$ Gly & 100 & $\begin{array}{l}\text { RND multidrug efflux } \\
\text { transporter }\end{array}$ \\
\hline & Del & 2585782 & $\mathrm{CCC}$ & $-C C$ & sll0058 (dnaK) & $\begin{array}{l}\text { Frameshift, stop } \\
\text { codon }\end{array}$ & 93 & $\begin{array}{l}\text { Heat-shock protein } \\
70 \text {, molecular chap- } \\
\text { erone }\end{array}$ \\
\hline & Del & 3430453 & $\mathrm{CTT}$ & $C-T$ & slr0756 (kaiA) & $\begin{array}{l}\text { Frameshift, stop } \\
\text { codon }\end{array}$ & 95 & $\begin{array}{l}\text { Circadian clock protein } \\
\text { KaiA homolog }\end{array}$ \\
\hline & Ins & 1372331 & CAA & CAAC & sll1165 (mutS) & $\begin{array}{l}\text { Frameshift, stop } \\
\text { codon }\end{array}$ & 80 & $\begin{array}{l}\text { DNA mismatch repair } \\
\text { protein MutS }\end{array}$ \\
\hline \multirow[t]{3}{*}{$\mathrm{T}(3)$} & Del & 1766850 & $\begin{array}{l}\text { ACCGCCCTTC- } \\
\text { CAACG }\end{array}$ & AC-----G & slr1963 (осp) & Del (Ala, Leu, Pro) & 88 & $\begin{array}{l}\text { Water-soluble carot- } \\
\text { enoid protein }\end{array}$ \\
\hline & SNP & 2271565 & $\mathrm{TGG}$ & TGA & hik43 & Stop codon & 98 & $\begin{array}{l}\text { Two-component } \\
\text { hybrid sensor and } \\
\text { regulator }\end{array}$ \\
\hline & Del & 2366401 & TCGGGGCGA & -------- & envD & Del (Ser, Gly, Arg) & 88 & $\begin{array}{l}\text { RND multidrug efflux } \\
\text { transporter }\end{array}$ \\
\hline \multirow[t]{2}{*}{$\mathrm{T}(4)$} & Ins & 492262 & TAT & TATT & $m c p A$ & $\begin{array}{l}\text { Frameshift, stop } \\
\text { codon }\end{array}$ & 95 & $\begin{array}{l}\text { Methyl-accepting } \\
\text { chemotaxis protein }\end{array}$ \\
\hline & SNP & 2366659 & GTT & $\mathrm{TTT}$ & envD & Val $\rightarrow$ Phe & 99 & $\begin{array}{l}\text { RND multidrug efflux } \\
\text { transporter }\end{array}$ \\
\hline
\end{tabular}

a Del, Ins, and SNP indicate an deletion, insertion, and single nucleotide polymorphism, respectively

b Mutation ratios indicates

$\mathrm{T}(2)$ and $\mathrm{T}(3)$ strains (Table 1), these mutations were also considered loss-of-function mutations. The $\Delta h i k 43$ strain showed the same growth phenotype as the $m c p A$ mutants in the presence of isobutanol (Fig. 4). Cell aggregation was also dispersed in the $\Delta h i k 43$ strain in the presence of $2 \mathrm{~g} / \mathrm{L}$ isobutanol (Fig. 2), indicating that hik43 also contributes to cell aggregation under alcohol stress.

$e n v D$ encodes the resistance nodulation division multidrug efflux transporter protein. Although the specific growth rate of the $\Delta e n v D$ strain decreased compared to the parental strain, in the presence of $2 \mathrm{~g} / \mathrm{L}$ isobutanol, the $\Delta e n v D$ strain grew in the presence of $5 \mathrm{~g} / \mathrm{L}$ isobutanol, which completely inhibits the growth of the parental strain (Fig. 4 and Additional file 1: Fig. S6). The specific growth rate of the $\Delta e n v D$ strain was lower than that of the evolved strain. The envD-R1, envD-R2, envD$\mathrm{R} 3$, and envD-R4 strains were constructed by introducing envD mutations derived from the $\mathrm{T}(1), \mathrm{T}(2), \mathrm{T}(3)$, and $\mathrm{T}(4)$ strains, respectively, into the $\Delta e n v D$ strain. Cell aggregation was observed in all envD mutants in the presence of $2 \mathrm{~g} / \mathrm{L}$ isobutanol as well as the parental strain (Fig. 2 and Additional file 1: Fig. S7). Because the envD$\mathrm{R} 1$, envD-R2, envD-R3, and envD-R4 strains showed a similar phenotype to the $\Delta e n v D$ strain (Additional file 1: 


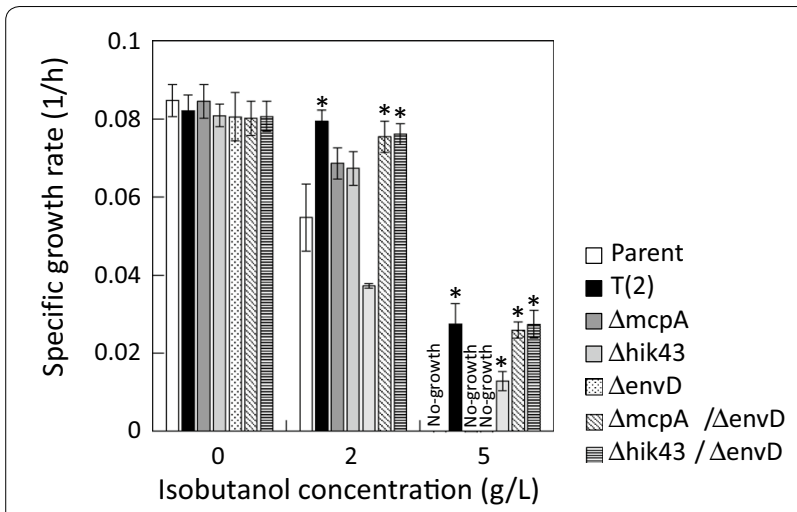

Fig. 4 Specific growth rates of the $\triangle m c p A / \triangle e n v D$ and $\triangle h i k 43 / \Delta e n v D$ strains under different concentrations of isobutanol. The asterisks indicate significant differences between the parental and the other strains ( $t$ test, $P<0.05$ ). The specific growth rate was calculated from an $\mathrm{OD}_{730}$ of 0 and $48 \mathrm{~h}$ of each batch culture. Error bars represent standard deviations of three replicates

Fig. S6), the envD mutations were also considered lossof-function mutations.

The specific growth rates of the constructed strains with single mutations of $m c p A$, hik43, and envD were lower than that of the evolved strains obtained from ALE experiments (Fig. 4). Because all four evolved strains possess combinatorial mutations of $m c p A$ and $e n v D$, or that of hik43 and envD (Fig. 3), it was assumed that the evolved strains acquired the isobutanol tolerance ability through the synergistic effect of these mutations. Since these $m c p A$, hik43, and envD mutations are considered loss-of-function mutations, the isobutanol tolerance of double deletion mutants $(\triangle m c p A / \Delta e n v D$ and $\Delta h i k 43 / \Delta e n v D$ strains) were evaluated. As expected, the $\Delta m c p A / \Delta e n v D$ and $\Delta h i k 43 / \Delta e n v D$ strains showed identical growth with the evolved strain under 0,2 , and $5 \mathrm{~g} / \mathrm{L}$ isobutanol concentrations (Fig. 4). Cell aggregation dispersed in $\triangle m c p A / \Delta e n v D$ and $\Delta h i k 43 / \Delta e n v D$ strains in the presence of $2 \mathrm{~g} / \mathrm{L}$ isobutanol (Fig. 2). These findings suggest that the evolved strains acquire isobutanol tolerance through the loss of function of $m c p A$ and $e n v D$, or that of hik43 and envD.

\section{Evaluation of stress tolerance against other alcohols of the evolved strains}

Cells acquiring stress tolerance exhibit a tolerance to the same type of stress $[18,19]$. Tolerance of the evolved strains against other alcohols such as ethanol, $n$-butanol, and isopentanol were evaluated (Fig. 5). The mutations important for alcohol tolerance were combinatorial malfunctions of $e n v D$ and $m c p A$, or that of $e n v D$ and hik43. Since both $\mathrm{T}(1)$ and $\mathrm{T}(4)$ strains have functional deficits in $m c p A$ and $e n v D$, and both $\mathrm{T}(2)$ and $\mathrm{T}(4)$ strains have functional deficits in hik43 and envD (Fig. 3), alcohol tolerances were evaluated for representative $T(1)$ and $T(2)$ strains. The specific growth rate of the evolved strains increased compared to the parental strain in the presence of $16 \mathrm{~g} / \mathrm{L}$ ethanol, which contains less carbon atoms than isobutanol. In the presence of 1 and $3 \mathrm{~g} / \mathrm{L} n$-butanol, which has the same number of carbon atoms of isobutanol with a different main chain, the specific growth rate of the evolved strains increased compared to the parental

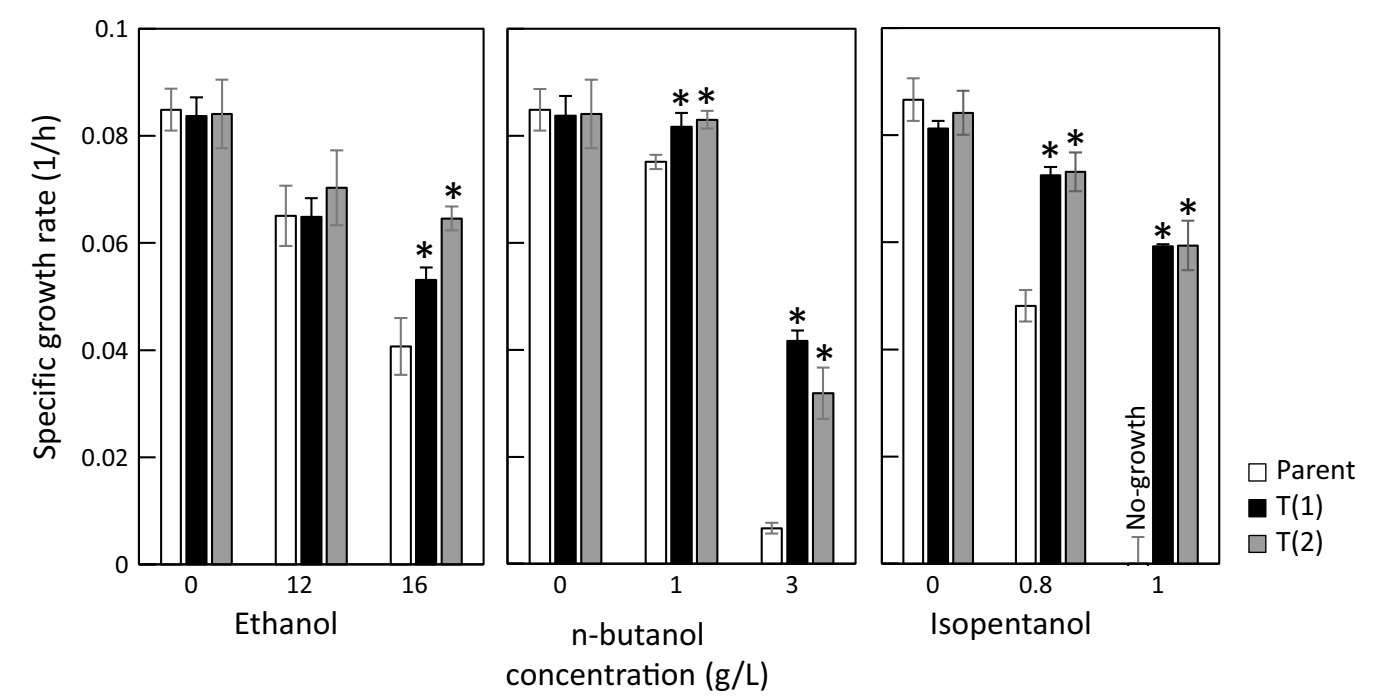

Fig. 5 Tolerances of the parental and the evolved strains against ethanol, n-butanol, and isopentanol. The asterisks indicate significant differences between the parental and the evolved strains ( $t$ test, $\mathrm{P}<0.05$ ). The specific growth rate was calculated from the $\mathrm{OD}_{730}$ of 0 and $48 \mathrm{~h}$ of each batch culture. Error bars represent standard deviations of three replicates 
strain. In the presence of 0.8 and $1 \mathrm{~g} / \mathrm{L}$ isopentanol, which contains more carbon atoms than isobutanol with the same main chain, the specific growth rate of the evolved strains also increased compared to the parental strain (Fig. 5). These findings suggest that the evolved strains obtained by ALE under isobutanol stress exhibited tolerance against isobutanol as well as ethanol, $n$-butanol, and isopentanol.

\section{Ethanol production using the evolved strain}

The evolved strains showed tolerance against not only isobutanol but also ethanol (Fig. 5). The effect of alcohol tolerance on ethanol production was investigated. Ethanol-producing strains of the parental and $\mathrm{T}(1)$ strains, called SE and SE-T(1) strains, were constructed by introducing heterologous genes for $p d c$ and adhII from Zymomonas mobilis. Culture profiles of SE and SE-T(1) strains are shown in Fig. 6. Although the specific growth rate of the SE-T(1) strain was slightly lower than that of the SE strain, enhanced ethanol production was observed in the SE-T(1) strain. The ethanol productivity at $72 \mathrm{~h}$ of SE and SE-T(1) strains were $10.4 \pm 0.4$ and $14.8 \pm 0.2 \mathrm{mg} \mathrm{L}^{-1}$ $\mathrm{OD}^{-1}$, respectively.

\section{Discussion}

In this study, evolved strains of Synechocystis sp. PCC 6803 were obtained using the ALE method in medium containing $2 \mathrm{~g} / \mathrm{L}$ isobutanol, to engineer a strain with strong tolerance against various alcohols (Fig. 5). Genome resequencing analysis demonstrated that the evolved strains possess mutations in $m c p A$ and envD, or hik43 and envD (Fig. 3). Reverse genetics approaches revealed that the mutations of $m c p A$, hik43, and $e n v D$ were loss-of-function mutations, and the evolved strains acquired resistance by combinatorial malfunctions of $e n v D$ and $m c p A$, or that of $e n v D$ and hik43 (Fig. 4).

In the $\triangle m c p A$ and $\Delta h i k 43$ strains, since no cell aggregation was observed in the presence of $2 \mathrm{~g} / \mathrm{L}$ isobutanol, $m c p A$ and $h i k 43$ genes are likely related to cell flocculation. It has been reported that deletion of mcpA or hik43 causes a loss of thick pili, which is essential for phototactic motility in Synechocystis sp. PCC 6803 [20]. Although the thick pili play a role in uptake of extracellular DNA in nature, these pili draw not only extracellular DNA but also the surrounding cells under isobutanol-induced stress. Cell aggregation of Synechocystis sp. PCC 6803 was also observed under $n$-butanol stress [22]. Transcriptome analysis demonstrated the up-regulation of genes involved in chemotaxis [6]. The relationship between cell flocculation size and ethanol tolerance has been investigated in yeast. Yeast has improved ethanol tolerance as flocculation size increases. On the other hand, in this study, alcohol tolerance was improved by making free of
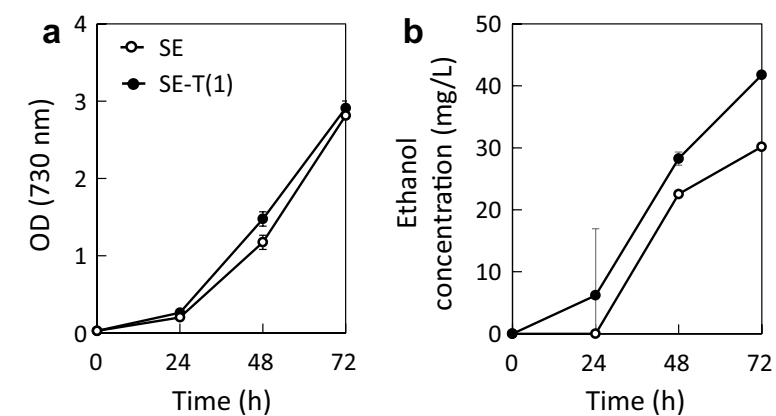

Fig. 6 Culture profiles of SE and SE-T(1) strains. a cell growth and b ethanol concentration

flocculation, so the tolerance was acquired by a mechanism different from yeast [23, 24].

The amino acid sequence of a protein encoding envD is similar to that of AcrB and AcrD of E. coli. Pairwise alignments show that both proteins share $38 \%$ amino acid sequence identity. Amino acid sequence similarities of the EnvD to the E. coli AcrB and AcrD are 58 and 59\%, respectively. E. coli AcrB and AcrF have been categorized into a same orthologous protein cluster of EnvD in CyanoClust for the protein homology database featuring cyanobacteria and plastids [25]. The AcrB is a component of the AcrAB-TolC transporter, which can export a broad variety of compounds with little chemical similarity [26]. It has been reported that the deletion of AcrAB-TolC contributed to enhance tolerance against solvents such as styrene, 1-octanol, and isobutanol [27]. Isobutanol stress causes leakage of quinone, an electron acceptor in the E. coli respiratory chain, owing to membrane damage [28]. Because AcrAB-TolC can also export quinone compounds [29] and is highly induced by isobutanol stress [28], the deletion of AcrAB-TolC increased isobutanol tolerance with reducing the quinone [18]. Although, to our knowledge, there are no studies on the molecular function of EnvD in Synechocystis sp. PCC 6803, its amino acid sequence similarity with AcrB would also be expected to involve quinone export. The envD deletion strain could acquire isobutanol tolerance with alleviation of quinone sink under the isobutanol stress condition. Despite the fact that the envD deletion makes it possible for the strain to grow in the presence of $5 \mathrm{~g} / \mathrm{L}$ isobutanol, the specific growth rate of the $e n v D$ deletion strain decreased compared to the parental strain in the presence of $2 \mathrm{~g} / \mathrm{L}$ isobutanol. Because deletion of membrane and membrane-associated proteins, such as transporters, would affect membrane lipids [27], the envD deletion negatively affected the cell growth indirectly in the presence of $2 \mathrm{~g} / \mathrm{L}$ isobutanol. 
Since the ALE experiments were conducted using $2 \mathrm{~g} / \mathrm{L}$ isobutanol, the mutants of $e n v D$ should not be selected because of its negative effect for the tolerance to this isobutanol level. Synergistic effects for isobutanol tolerance induced by the combinatorial gene deletions of $e n v D$ and $m c p A$, or that of $e n v D$ and $h i k 43$ are shown in Fig. 4. The findings indicate that first the mutations in $m c p A$ or hik43 were introduced, followed by the mutations in $e n v D$, since non-aggregated cells by $m c p A$ or hik43 mutation are easy to subculture in the passaged cultures. Although the mechanisms of the synergistic effect for isobutanol tolerance by these two genes are still unclear, photon supply to each cell increases through interference with cell aggregation. Additionally, the metabolic activities could be enhanced through alleviation of quinone sink with envD mutations. It is difficult to rationally predict such synergistic effects for tolerance by multiple gene deletions. The ALE method has become a powerful tool for strain improvement to obtain stress tolerance.

The expression of small heat-shock protein HspA (sll1514) and hypothetical protein CccS (slr1617) has improved 1-butanol tolerance [6]. Furthermore, it is known that a response regulator (Slr1037) is involved in butanol tolerance of Synechocystis sp. PCC 6803 [7], and co-overexpression of slr1037 and sll0039 improves butanol tolerance by $133 \%$ [9]. An overexpression of SigB is also known to improve 1-butanol tolerance in Synechocystis sp. PCC 6803 [10]. By understanding the mechanism of the alcohol tolerance acquisition by the combinatorial malfunctions of $e n v D$ and $m c p A$, or that of $e n v D$ and $h i k 43$, it may help to further enhance the alcohol tolerance in coordination with these previous findings.

The SE-T(2) strain, in which ethanol-producing genes were introduced, shows enhanced ethanol production compared to the control strain. This indicates that the ethanol tolerance ability is effective for enhancing the ethanol production rate. Since ethanol affected the growth of the parental strain at concentrations of up to $16 \mathrm{~g} / \mathrm{L}$ (Fig. 5), a higher ethanol concentration was required to inhibit growth of the SE-T(2) strain $(28.3 \mathrm{mg} / \mathrm{L}$ at $48 \mathrm{~h})$. If ethanol excretion is a rate-limiting step, the ethanol concentration in the cells becomes much higher than that of the extracellular culture broth. Therefore, the ethanol-producing cells would be exposed to a stress condition in which the concentration of ethanol is higher than the produced ethanol concentration. Furthermore, the transporter malfunction may indirectly contribute to ethanol production. Because the mechanism is unknown, detailed metabolic analysis is necessary to clarify this.

In this study, we chose ethanol, which has been widely studied in cyanobacterial bio-production as a target alcohol. Since the evolved strains showed tolerance abilities against various alcohols (Fig. 5), the strains have the potential to enhance the production rate of a wide variety of alcohols. Since the mutation for enhancing alcohol tolerance was obtained by ALE experiments using isobutanol, it is expected to contribute in isobutanol productions, especially. Isobutanol showing more strong toxicity may clearly demonstrate the effect of the mutations.

\section{Conclusions}

Alcohol stress tolerant strains of Synechocystis sp. PCC 6803 were obtained via the ALE method using medium containing isobutanol, which induces strong toxicity. The evolved strains showed increased growth rates in more than $2 \mathrm{~g} / \mathrm{L}$ isobutanol, and could grow in the presence of $5 \mathrm{~g} / \mathrm{L}$ isobutanol, which completely inhibits the growth of the parental strain. Genome resequencing and reverse genetics analyses revealed that the evolved strains acquired their resistive capacity owing to combinatorial malfunctions of $e n v D$ and $m c p A$, or that of $e n v D$ and $h i k 43$. Furthermore, the evolved strains also demonstrated tolerance against a wide variety of alcohols, such as ethanol, $n$-butanol, and isopentanol. The ethanol productivity successfully increased to $142 \%$ of the control strain by introducing an ethanol-producing pathway into the evolved strain.

\section{Methods}

\section{Strain and culture condition}

The Synechocystis sp. PCC 6803 glucose-tolerant strain served as the parent strain. All strains used in this study are listed in Table 1. Cultures were performed autotrophically using 100-mL Erlenmeyer flasks with $20 \mathrm{~mL}$ BG11 medium [30] at $34{ }^{\circ} \mathrm{C}$, with rotary agitation at $150 \mathrm{rpm}$ (BR-43FL shaker; TAITEC Co., Ltd., Japan) under continuous illumination $\left(40 \mu \mathrm{mol} \mathrm{m} \mathrm{m}^{-2} \mathrm{~s}^{-1}\right)$ by white light-emitting diodes (LC-LED450 W, TAITEC Co., Ltd., Japan). The BG11 medium was supplemented with $50 \mathrm{mM}$ $\mathrm{NaHCO}_{3}$ and the required antibiotics.

\section{Analytical methods}

Cell growth was measured as optical density at $730 \mathrm{~nm}$ $\left(\mathrm{OD}_{730}\right)$ using a spectrophotometer (UVmini-1240, Shimadzu, Kyoto, Japan). The ethanol concentration of the culture was measured by gas chromatography with a hydrogen flame ionization detector (7890A; Agilent Technologies, CA, USA) and a Stabiliwax column (0.32 mm internal diameter, $60 \mathrm{~m}$ length, and $1 \mu \mathrm{m}$ thickness; Restek, PA, USA). The analytical conditions are described elsewhere [31]. A light optical microscope (Imager M2, Carl Zeiss, Jena, Germany) with AxioVision Release 4.8 was used to examine the flocculation of the cells. 


\section{Tolerance test of alcohols}

Cells were inoculated at $\mathrm{OD}_{730}$ of 0.05 for pre-culture, and then incubated in the above conditions. After 3 days, the pre-cultivated cells were harvested by centrifugation, and then inoculated into the fresh BG11 medium at $\mathrm{OD}_{730}$ of 0.03 . To test the alcohol tolerance, an appropriate amount of $99 \%(\mathrm{w} / \mathrm{w})$ isobutanol, $99.5 \%(\mathrm{w} / \mathrm{w})$ ethanol, $99 \%(\mathrm{w} / \mathrm{w}) n$-butanol, or $98 \%(\mathrm{w} / \mathrm{w})$ isopentanol was supplemented into the medium. A silicon cap was used in the culture to prevent evaporation of alcohols from the culture broth. The specific growth rate was calculated from $\mathrm{OD}_{730}$ during the exponential growth phase (0-48 h).

\section{Adaptive laboratory evolution}

Sequential serial passages were performed using the parental strain and BG11 medium with $2 \mathrm{~g} / \mathrm{L}$ isobutanol under the previously mentioned culture conditions. Cells were inoculated into fresh medium every 3.5 days at $\mathrm{OD}_{730}$ of 0.03 to maintain the growth phase in four parallel cultures. A silicon cap was used to prevent the evaporation of isobutanol. The specific growth rate was calculated from $\mathrm{OD}_{730}$ up to $0-48 \mathrm{~h}$ for each culture. After the final cultivation, cells were stored at $-80{ }^{\circ} \mathrm{C}$ in a $15 \%$ glycerol solution. The freeze stock was streaked on a BG11 plate for single colony isolation. The obtained single colonies were inoculated in BG11 medium, and cultured with $2 \mathrm{~g} / \mathrm{L}$ isobutanol. The strains were stored at $-80{ }^{\circ} \mathrm{C}$ in a $15 \%$ glycerol solution, and labeled as $\mathrm{T}(1)$, $\mathrm{T}(2), \mathrm{T}(3)$, and $\mathrm{T}(4)$.

\section{Whole genome sequencing}

The genomic DNAs of the parental and its evolved strains were extracted using a genome extraction kit (Qiagen, CA, USA). The DNA samples were sequenced using a MiSeq sequencer (Illumina) with the MiSeq Reagent kit v2 (Illumina) generating $150 \mathrm{bp}$ paired-end reads. The sequenced reads were mapped to the genome sequence of the Synechocystis sp. PCC 6803 GT-I strain (NCBI Reference Sequence No.: NC_017038.1) [16] using the Bowtie 2 software ver. 2.2.3 [32]. The SNPs of the parental strain to the reference genome were identified using SAMtools ver 1.0. The SNPs of the evolved strains were also checked using the Integrative Genomics Viewer [33]. The SNPs of the evolved strains identified by whole genome sequencing were confirmed by Sanger sequencing (Eurofins) using DNA fragments, including the mutation site amplified by PCR with the primer pair listed in Additional file 1: Table S1.

\section{Genomic manipulations}

Strains used in this study are listed in Table 2. Single gene deletion strains of $\Delta m c p A, \Delta h i k 43$, and $\Delta e n v D$ were
Table 2 Strains used in this study

\begin{tabular}{|c|c|c|}
\hline Strains & Description & References \\
\hline Parent & Synechocystis sp. PCC 6803 GT-I & {$[31]$} \\
\hline$T(1)$ & Obtained from ALE in No. 1 & This work \\
\hline $\mathrm{T}(2)$ & Obtained from ALE in No. 2 & This work \\
\hline $\mathrm{T}(3)$ & Obtained from ALE in No. 3 & This work \\
\hline $\mathrm{T}(4)$ & Obtained from ALE in No. 4 & This work \\
\hline$\triangle m c p A$ & $m c p A:: \mathrm{Km}^{\mathrm{r}}$ & This work \\
\hline$\Delta h i k 43$ & hik43:: $\mathrm{Km}^{r}$ & This work \\
\hline$\triangle e n v D$ & envD::Km ${ }^{r}$ & This work \\
\hline mcpA-R1 & $m c p A:: m c p A T(1) S m^{r}$ & This work \\
\hline mcpA-R4 & $m c p A:: m c p A T(2) S m^{r}$ & This work \\
\hline envD-R1 & envD::envDT(1) Sm ${ }^{r}$ & This work \\
\hline envD-R2 & envD::envDT(2) Smr & This work \\
\hline envD-R3 & envD::envDT(3) Sm ${ }^{r}$ & This work \\
\hline envD-R4 & envD::envDT(4) $\mathrm{Sm}^{r}$ & This work \\
\hline$\triangle e n v D / \Delta m c p A$ & envD:::Km${ }^{r}, m c p A:: \mathrm{Sm}^{r}$ & This work \\
\hline$\Delta e n v D / \Delta h i k 43$ & 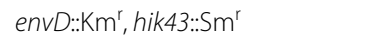 & This work \\
\hline SE & $n d h B:: P_{p s b A 2} p d c a d h / l \mathrm{Am}^{r}$ & {$[31]$} \\
\hline SE-T(1) & $\mathrm{T}(1) n d h B:: \mathrm{P}_{\mathrm{psbA2}} p d c$ adh $/ / \mathrm{Am}^{r}$ & This work \\
\hline
\end{tabular}

constructed using Synechocystis sp. PCC 6803 glucosetolerant strain as the parental strain by replacing each corresponding gene with a kanamycin-resistance gene. To construct strains in which mutations were identified after ALE, the kanamycin-resistance gene in the corresponding gene deletion strain was replaced with the mutated gene and a streptomycin-resistance gene. Double gene deletion strains of $\triangle m c p A / \Delta e n v D$ and $\Delta h i k 43 / \Delta e n v D$ were constructed using the $\Delta e n v D$ strain by replacing $m c p A$ or $h i k 43$ with a streptomycin-resistance gene. Complete segregation of the target site by the antibiotic resistance gene was confirmed by the disparity in the sizes of the PCR products generated using the primers that bound outside the upstream and downstream regions of target site for homologous recombination. Detailed procedures for strain construction, including plasmid and primer information, are described in an additional document.

\section{Additional file}

Additional file 1. Additional figures, table, and details procedures for strain construction.

Authors' contributions

All authors contributed to the planning of this study. TM and KY performed experiments. TM, YT, and HS drafted the manuscript. All authors read and approved the final manuscript.

Acknowledgements

We thank Dr. Yuu Hirose for whole genome sequencing of the parental, $T(1)$, $\mathrm{T}(2), \mathrm{T}(3)$, and $\mathrm{T}(4)$ strains. 


\section{Competing interests}

The authors declare that they have no competing interests.

\section{Availability of supporting data}

The whole genome sequence data of $T(1), T(2), T(3)$, and $T(4)$ strains are deposited in the DNA Data Bank of Japan (DDBJ) Sequence Read Archive (DRA) under the Accession Number DRA006219.

\section{Consent for publication}

Not applicable.

\section{Ethics approval and consent to participate}

Not applicable.

\section{Funding}

This work was supported by JSPS KAKENHI Grant Number 16H06559. This work was also supported by the Grant in Aid for Scientific Research (A) No. 24246134.

\section{Publisher's Note}

Springer Nature remains neutral with regard to jurisdictional claims in published maps and institutional affiliations.

Received: 29 September 2017 Accepted: 11 December 2017

Published online: 20 December 2017

\section{References}

1. Dexter J, Armshaw P, Sheahan C, Pembroke JT. The state of autotrophic ethanol production in Cyanobacteria. J Appl Microbiol. 2015;119:11-24.

2. Atsumi S, Higashide W, Liao JC. Direct photosynthetic recycling of carbon dioxide to isobutyraldehyde. Nat Biotechnol. 2009;27:1177-80.

3. Smith KM, Liao JC. An evolutionary strategy for isobutanol production strain development in Escherichia coli. Metab Eng. 2011;13:674-81.

4. Yamamoto S, Suda M, Niimi S, Inui M, Yukawa H. Strain optimization for efficient isobutanol production using Corynebacterium glutamicum under oxygen deprivation. Biotechnol Bioeng. 2013;110:2938-48.

5. Varman AM, Xiao Y, Pakrasi HB, Tang YJ. Metabolic engineering of Synechocystis sp. strain PCC 6803 for isobutanol production. Appl Environ Microbiol. 2013;79:908-14.

6. Anfelt J, Hallström B, Nielsen J, Uhlén M, Hudson EP. Using transcriptomics to improve butanol tolerance of Synechocystis sp. strain PCC 6803. Appl Environ Microbiol. 2013;79:7419-27.

7. Chen L, Wu L, Wang J, Zhang W. Butanol tolerance regulated by a twocomponent response regulator SIr 1037 in photosynthetic Synechocystis sp. PCC 6803. Biotechnol Biofuels. 2014;7:89.

8. Niu X, Zhu Y, Pei G, Wu L, Chen L, Zhang W. Elucidating butanol tolerance mediated by a response regulator SII0039 in Synechocystis sp. PCC 6803 using a metabolomic approach. Appl Microbiol Biotechnol. 2015:99(4):1845-57.

9. Gao X, Sun T, Wu L, Chen L, Zhang W. Co-overexpression of response regulator genes s/r1037 and sll0039 improves tolerance of Synechocystis sp. PCC 6803 to 1-butanol. Bioresour Technol. 2017;245(Pt B):1476-83.

10. Kaczmarzyk D, Anfelt J, Särnegrim A, Hudson EP. Overexpression of sigma factor SigB improves temperature and butanol tolerance of Synechocystis sp. PCC6803. J Biotechnol. 2014:182-183:54-60.

11. Sandberg TE, Pedersen M, LaCroix RA, Ebrahim A, Bonde M, Herrgard MJ Palsson $\mathrm{B} \varnothing$, Sommer M, Feist AM. Evolution of Escherichia coli to $42^{\circ} \mathrm{C}$ and subsequent genetic engineering reveals adaptive mechanisms and novel mutations. Mol Biol Evol. 2014;31:2647-62.

12. LaCroix RA, Sandberg TE, O'Brien EJ, Utrilla J, Ebrahim A, Guzman Gl, Szubin $R$, Palsson B $\varnothing$, Feist AM. Use of adaptive laboratory evolution to discover key mutations enabling rapid growth of Escherichia col K-12 MG1655 on glucose minimal medium. Appl Environ Microbiol. 2015;81:17-30.
13. Dragosits M, Mattanovich D. Adaptive laboratory evolution—principles and applications for biotechnology. Microb Cell Fact. 2013;12:64.

14. Lee $\mathrm{DH}$, Feist $\mathrm{AM}$, Barrett $\mathrm{CL}$, Palsson $\mathrm{B} \varnothing$. Cumulative number of cell divisions as a meaningful timescale for adaptive laboratory evolution of Escherichia coli. PLoS ONE. 2011:6:e26172.

15. Kaneko T, Sato S, Kotani H, Tanaka A, Asamizu E, Nakamura Y, Miyajima N, Hirosawa M, Sugiura M, Sasamoto S, Kimura T, Hosouchi T, Matsuno A, Muraki A, Nakazaki N, Naruo K, Okumura S, Shimpo S, Takeuchi C, Wada T, Watanabe A, Yamada M, Yasuda M, Tabata S. Sequence analysis of the genome of the unicellular cyanobacterium Synechocystis sp. strain PCC6803. II. Sequence determination of the entire genome and assignment of potential protein-coding regions. DNA Res. 1996;3:109-36.

16. Kanesaki Y, Shiwa Y, Tajima N, Suzuki M, Watanabe S, Sato N, Ikeuchi M, Yoshikawa $\mathrm{H}$. Identification of substrain-specific mutations by massively parallel whole-genome resequencing of Synechocystis sp. PCC 6803. DNA Res. 2012;19:67-79.

17. Uchiyama J, Kanesaki Y, Iwata N, Asakura R, Funamizu K, Tasaki R, Agatsuma M, Tahara H, Matsuhashi A, Yoshikawa H, Ogawa S, Ohta H. Genomic analysis of parallel-evolved cyanobacterium Synechocystis sp. PCC 6803 under acid stress. Photosynth Res. 2015;125:243-54.

18. Atsumi S, Wu TY, Machado IM, Huang WC, Chen PY, Pellegrini M, Liao JC. Evolution, genomic analysis, and reconstruction of isobutanol tolerance in Escherichia coli. Mol Syst Biol. 2010;6:449.

19. Suzuki S, Horinouchi T, Furusawa C. Prediction of antibiotic resistance by gene expression profiles. Nat Commun. 2014;5:5792.

20. Yoshihara S, Geng X. Ikeuchi M. pilG gene cluster and split pilL genes involved in pilus biogenesis, motility and genetic transformation in the cyanobacterium Synechocystis sp. PCC 6803. Plant Cell Physiol. 2002:43:513-21.

21. Pisareva T, Shumskaya M, Maddalo G, Ilag L, Norling B. Proteomics of Synechocystis sp. PCC 6803. Identification of novel integral plasma membrane proteins. FEBS J. 2007;274:791-804.

22. Tian $X$, Chen L, Wang J, Oiao J, Zhang W. Quantitative proteomics reveals dynamic responses of Synechocystis sp. PCC 6803 to next-generation biofuel butanol. J Proteom. 2013:78:326-45.

23. Lei J, Zhao X, Ge X, Bai F. Ethanol tolerance and the variation of plasma membrane composition of yeast floc populations with different size distribution. J Biotechnol. 2007:131:270-5.

24. Cheng C, Zhang M, Xue C, Bai F, Zhao X. Development of stress tolerant Saccharomyces cerevisiae strains by metabolic engineering: new aspects from cell flocculation and zinc supplementation. J Biosci Bioeng. 2017:123:141-6.

25. Sasaki NV, Sato N. CyanoClust: comparative genome resources of cyanobacteria and plastids. Database (Oxford). 2010;2010:bap025

26. Du D, Wang Z, James NR, Voss JE, Klimont E, Ohene-Agyei T, Venter H, Chiu W, Luisi BF. Structure of the AcrAB-TolC multidrug efflux pump. Nature. 2014;509:512-5.

27. Mukhopadhyay A. Tolerance engineering in bacteria for the production of advanced biofuels and chemicals. Trends Microbiol. 2015:23:498-508.

28. Brynildsen MP, Liao JC. An integrated network approach identifies the isobutanol response network of Escherichia coli. Mol Syst Biol. 2009;5:277.

29. Shyu JB, Lies DP, Newman DK. Protective role of tolC in efflux of the electron shuttle anthraquinone-2,6-disulfonate. J Bacteriol. 2002;184:1806-10.

30. Yoshikawa K, Hirasawa T, Ogawa K, Hidaka Y, Nakajima T, Furusawa C, Shimizu $\mathrm{H}$. Integrated transcriptomic and metabolomic analysis of the central metabolism of Synechocystis sp. PCC 6803 under different trophic conditions. Biotechnol J. 2013:8:571-80.

31. Yoshikawa K, Hirasawa T, Shimizu H. Effect of malic enzyme on ethanol production by Synechocystis sp. PCC 6803. J Biosci Bioeng. 2015;119:82-4

32. Langmead B, Salzberg SL. Fast gapped-read alignment with Bowtie 2. Nat Methods. 2012:9:357-9.

33. Robinson JT, Thorvaldsdóttir H, Winckler W, Guttman M, Lander ES, Getz G, Mesirov JP. Integrative genomics viewer. Nat Biotechnol. 2011;29:24-6. 